\title{
On the radio/X-ray correlation in microquasars
}

\author{
Y. Q. Xue and W. Cui
}

Department of Physics, Purdue University, West Lafayette, IN 47907, USA

e-mail: [xuey; cui]@physics.purdue.edu

Received 22 November 2006 / Accepted 4 February 2007

\begin{abstract}
Context. The broadband spectral energy distribution of microquasars has proven to be a valuable tool for assessing the roles of jets and accretion flows in microquasars, as well as the coupling between the two. The coupling might manifest itself observationally in the correlated radio and X-ray variabilities of a source. Such a radio/X-ray correlation has indeed been seen in several microquasars during the low-hard state and subsequently been claimed to be universal for all. If proven, the universal correlation would have profound implications on theoretical models. However, there is already observational evidence that suggests otherwise.

Aims. In this paper, we critically examine the radio/X-ray correlation in a sample of microquasars, in the low-hard state as well as during state transitions, with a goal of testing the claimed universality of the correlation on observational grounds.

Methods. We have assembled a comprehensive data set from the simultaneous/contemporaneous radio and X-ray observations of representative microquasars. The data have allowed us to quantify the radio/X-ray correlation on a source by source basis.

Results. We find that the radio/X-ray correlation of microquasars exhibits diverse behaviors, both in the low-hard and transitional states, ranging from being very week (or none at all) to very strong. There is even a hint of spectral dependence of the correlation in some cases.

Conclusions. Our results rule out the claimed universality of the radio/X-ray correlation of microquasars, even for the low-hard state. On the other hand, we do find that the radio and X-ray variabilities are, to varying degrees, correlated in most cases.
\end{abstract}

Key words. accretion, accretion disks - black hole physics - stars: general - X-rays: binaries

\section{Introduction}

Microquasars are inherently broadband objects, like active galactic nuclei and gamma-ray bursts. Roughly speaking, the spectral energy distribution (SED) of microquasars can be divided into three main regimes (see review by, e.g., Liang 1998): a power-law distribution at radio frequencies, which could extend up to infrared/optical regions (e.g., Chaty et al. 2003), a blackbody-like profile in the middle (from optical to soft $\mathrm{X}$-ray frequencies), and a power-law distribution again at hard $\mathrm{X}$-ray/soft gamma-ray energies (which eventually rolls over). To interpret the SED of microquasars, two classes of models have been proposed, with one being accretion-based (e.g., Esin et al. 2001) and the other jet-based (Markoff et al. 2001, 2003). However, neither class of models can satisfactorily explain the overall SED of microquasars. The accretion-based models typically fail at low frequencies (radio and infrared in particular; see discussion in Yuan et al. 2005), while the jet-based models suffer from a number of issues (Zdziarski et al. 2003). A hybrid scenario seems more likely (and natural), in which both the accretion flows and jets make critical contributions to the SED of microquasars but the relative importance of the contributions may vary at different frequencies. For example, the coupled accretion-jet model proposed by Yuan et al. (2005) postulates that the radio (and a bulk of infrared) emission comes entirely from the jets and the X-ray (and soft gamma-ray) emission is due almost entirely to the accretion flows. An effective way to assess the roles of the jets and accretion flows in microquasars is, therefore, to study the broadband SED of these systems.

A key ingredient in nearly all models on jet formation is the physical coupling between the jets and accretion flows (e.g., Falcke \& Biermann 1995; Meier 2001). For microquasars, such a jet-accretion coupling might manifest itself in the correlated radio and X-ray variabilities (Robertson \& Leiter 2004; Yuan $\&$ Cui 2005). Indeed, a positive radio/X-ray correlation was first established for GX 339-4 in the low-hard state (Corbel et al. 2003). A similar correlation was subsequently also seen in V404 Cyg (also in the low-hard state), which led to the claim that the correlation is the same for all microquasars (Gallo et al. 2003). If proven, such a universal radio/X-ray correlation would have profound implications on theoretical models (e.g., Heinz 2004; Robertson \& Leiter 2004; Yuan \& Cui 2005). However, as noted by Xue et al. (2006), the data shown by Gallo et al. (2003) are dominated by only two sources (GX 339-4 and V404 Cyg); the data points for other sources are very limited in their dynamical ranges yet already show significant deviations from the universal correlation. Subsequent studies provided additional evidence for such deviations (see, e.g., Fig. 5 in Choudhury et al. 2003; and Fig. 17 in Wilms et al. 2006), even for the low-hard state, although the authors tended to be dismissive of the discrepancies. Based on these and other observations, Xue et al. (2006) suggested that, while the radio and X-ray variabilities could well be correlated among microquasars individually, the correlation might not be quantitatively the same for all.

To resolve this important issue, we have undertaken an effort to systematically examine the simultaneous radio and X-ray data already taken for a representative sample of microquasars. We believe that the best way to separate personality from universality is to carefully examine the radio/X-ray correlation on a source by source basis. We have also taken the opportunity to examine the radio/X-ray correlation beyond the low-hard state, to the extent that the data allow. 
Table 1. Source sample.

\begin{tabular}{lccc}
\hline \hline $\begin{array}{l}\text { Source } \\
\text { name }\end{array}$ & ${\text { Year(s })^{a}}^{a}$ & ${\text { State }(\mathrm{s})^{b}}^{b}$ & $\begin{array}{l}\text { Radio data } \\
\text { references }\end{array}$ \\
\hline Cygnus X-1 & 1999 & LHS & 1 \\
GRO J1655-40 & 2005 & LHS, TS & 2 \\
GX 339-4 & $1997-1999,2002$ & LHS, TS & 3,4 \\
H 1743-322 & 2003 & LHS, TS & $5-8$ \\
V4641 Sgr & $1999,2002-2004$ & LHS, TS & $9-16$ \\
XTE J1118+480 & 2000,2005 & LHS & $17-19$ \\
XTE J1859+226 & 1999 & TS & 20 \\
\hline
\end{tabular}

References: (1) Gleissner et al. (2004); (2) Brocksopp et al. (2005);

(3) Corbel et al. (2000); (4) Gallo et al. (2004); (5) Rupen et al. (2003a); (6) Rupen et al. (2003b); (7) Rupen et al. (2003c); (8) Kalemci et al. (2006); (9) Ishwara-Chandra \& Pramesh (2005); (10) Uemura et al. (2004); (11) Rupen et al. (2004); (12) Rupen et al. (2003d); (13) Rupen et al. (2003); (14) Senkbeil \& Sault (2004); (15) Rupen et al. (2004); (16) Hjellming et al. (2000); (17) Fender et al. (2001); (18) Dhawan et al. (2000); (19) Rupen et al. (2005); (20) Brocksopp et al. (2002).

Note: ${ }^{a}$ The year(s) in which the data were taken. ${ }^{b}$ The spectral states covered by the data. LHS stands for "low-hard state" and TS "transitional state".

\section{Data}

We first carried out a comprehensive search for radio observations of microquasars in the archival databases and in the literatures. For each radio observation found, we then checked the availability of simultaneous/contemporaneous X-ray data on the same source. For this work, we used only data sets in which the corresponding radio and X-ray observations were taken within one day of each other. In the end, we found over 300 sets of the radio/X-ray observations that satisfy our criteria. Of the total, about $25 \%$ contain the radio and X-ray observations taken simultaneously, about $50 \%$ observations taken within $5 \mathrm{~h}$ of each other, and about $88 \%$ observations taken within $12 \mathrm{~h}$ of each other.

\subsection{Radio data}

Table 1 summarizes the sources of the radio data used in this work, as well as the year(s) in which the data were taken for each microquasar in the sample. The data come from observations made with a number of radio observatories, including the Very Large Array, the Australia Telescope Compact Array, the Molonglo Observatory Synthesis Telescope, the Ryle Telescope, the Multi-Element Radio-Linked Interferometer Network, the Giant Metrewave Radio Telescope, and the Green Bank Interferometer, and, in most cases, at more than one frequency (see the listed references for details on the observations).

\subsection{X-ray data}

The X-ray data were derived exclusively from the Rossi X-ray Timing Explorer (RXTE) archival database. For this work, we used only data from the Proportional Counter Array (PCA). The PCA consists of five nearly identical proportional counter units (PCUs) but not all of the PCUs are always turned on for a given observation. For the observations of interest here, PCU 2 was always in operation. For simplicity, we used only the PCU 2 data, which already provide sufficient statistics for our purposes.

The RXTE data were reduced with FTOOLS 6.0.1 (Blackburn 1995), following the usual procedure that we have adopted (see, e.g., Cui 2004; Xue \& Cui 2005). For each observation, we first filtered data by following the standard procedure for bright sources, which resulted in a list of good time intervals (GTIs). We then simulated background events for the observation with the latest background model that is appropriate for bright sources (pca_bkgd_cmbrightvle_eMv20051128.mdl) ${ }^{1}$. Using the GTIs, we then produced the PCU 2 spectrum from the Standard2 data for the observation. Note that we used only data from the first xenon layer, which is best calibrated. We repeated the steps to derive a corresponding background spectrum for PCU 2 from the simulated background events.

We carried out the spectral modeling in XSPEC 12.2.0ba (Arnaud 1996). Because the RXTE data have poor signal-tonoise ratios at low energies, during the spectral fitting we fixed the hydrogen column density for each source at the Galactic value along the line of sight (Dickey \& Lockman 1990). Also, because the main purposes of the spectral modeling here is to quantify the shape of the SED and derive accurate X-ray fluxes, we limited ourselves to simple empirical models, including power law and power law plus multi-color disk ("diskbb" in XSPEC), with or without a high-energy rollover ("highecut" in XSPEC). In some cases, we also needed an additional Gaussian component and sometimes an absorption edge ("smedge" in XSPEC) to account for the residuals. A discussion of the physical nature of each component in the adopted models is beyond the scope of this work. We limited the spectral fits to an energy range of $3-30 \mathrm{keV}$ (which is nominally covered by the first xenon layer) and added $1 \%$ systematic uncertainty to the data. Statistically acceptable fits (i.e., with the reduced $\chi^{2}$ values around unity) were obtained for all observations. In each case, the SED was then derived by using the best-fit model to unfold and de-absorb the measured spectrum.

\section{Results}

To facilitate direct comparisons with the published results (e.g., in Gallo et al. 2003), we adopted the energy band of $2-11 \mathrm{keV}$ for computing X-ray fluxes. The X-ray fluxes presented in this work have all been corrected for interstellar absorption.

\subsection{Diversity of the radio/X-ray correlation in microquasars}

Figure 1 summarizes the diverse behaviors of the radio/X-ray correlation that we found among individual microquasars. In the figure, the radio flux density $\left(F_{\mathrm{R}}\right)$ and the X-ray flux $\left(F_{\mathrm{X}}\right)$ are shown for four selected microquasars in our sample that are all in the low-hard state.

Cyg X-1 is representative of persistent microquasars (which are in the minority of the population). It is perhaps still the most frequently observed and studied member of the class. A tremendous amount of X-ray data have been collected on Cyg X-1. For this work, we selected data from the RXTE observations of the source in 1999, because of the large dynamical range of the radio data (Gleissener et al. 2004). The results show that, while there seems to be a general positive correlation between the radio and $\mathrm{X}$-ray variabilities of the source, the correlation is a fairly loose one. This is in general agreement with the conclusion of Wilms et al. (2006) based on some of the same data (see their Fig. 17). Fitting the data with a power law we found $F_{\mathrm{R}} \propto F_{\mathrm{X}}^{0.5 \pm 0.4}$. Obviously, the fit is very poor, as shown in Fig. 1(1). For comparison, Wilms et al. found $F_{\mathrm{R}} \propto F_{\mathrm{X}}^{1.05}$ when they included additonal low-hard state data in their analysis. They speculated that

\footnotetext{
1 The only exception is XTE J1118+480 in its 2005 outburst, in which the source was quite faint and we had to use the background model for faint sources (pca_bkgd_cmfaintl7_eMv20051128.mdl).
} 


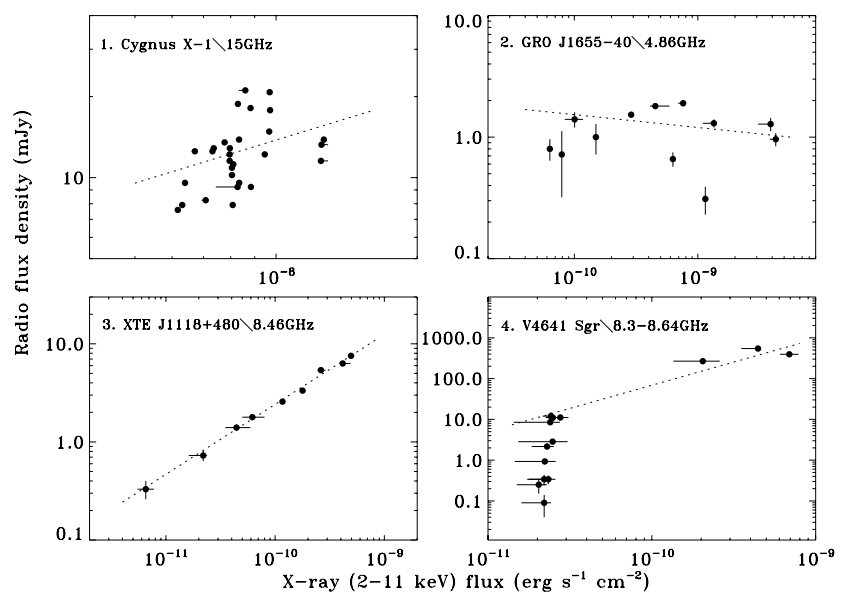

Fig. 1. The observed radio/X-ray correlations in the selected microquasars. Only data for the low-hard state are shown. The dotted line shows the best power-law fit to the data. Note, however, that in the case of V4641 Sgr the fit was made only to the seven data points (two of which are too close to be seen separately) at high fluxes.

the observed deviation from the universal radio/X-ray correlation might be related to the fact that Cyg $\mathrm{X}-1$ is a persistent source. However, if the radio/X-ray correlation is related to the coupling of the jets and accretion flows, it would not be obvious why it has anything to do with a source being persistent or transient.

Together with GRS 1915+105, GRO J1655-40 is among the first microquasars whose jets are seen to exhibit superluminal motions (Mirabel \& Rodriguez 1999). In fact, the study of these sources led to the use of the term "microquasar" in the literatures, highlighting their similarities to supermassive black hole systems. GRO J1655-40 is representative of transient microquasars (which is the dominant majority). After spending many years in quiescence, it underwent a major outburst in 2005, during which it became the target of intensive monitoring efforts both in the radio and X-ray wavebands. Some of the results based on those observations have been reported by Shaposhnikov et al. (2007), including the radio/X-ray correlation. We re-analyzed the data here for two reasons. One is to compare directly with other sources in the sample, highlighting the diverse behaviors, and the other is to examine the possible spectral-dependence of the radio/X-ray correlation (see the next section). Confirming the results of Shaposhnikov et al. (2007), Fig. 1(2) shows that the source varied greatly in X-rays in the low-hard state but hardly varied at all in radio. Fitting the data with a power law, we found $F_{\mathrm{R}} \propto F_{\mathrm{X}}^{-0.11 \pm 0.04}$. Note that the fit is not statistically acceptable in this case either. This result shows the most dramatic departure from the claimed universal radio/X-ray correlation.

XTE J1118+480 was the focus of multiple intensive multiwavelength campaigns during its outburst in 2000 (e.g., Hynes et al. 2000; McClintock et al. 2001; Frontera et al. 2001; Chaty et al. 2003). Intriguingly, it remained in the low-hard state throughout the outburst, which makes it representative of a small group of transient microquasars (including, e.g., V404 Cyg and GRO J0422+32) that are known to do the same. Unfortunately, the dynamical range of the simultaneous radio and X-ray observations is too limited during the outburst, which is hardly adequate for investigating radio/X-ray correlations. Luckily, the source was seen in outburst again in 2005. It was intensely followed during this outburst both in the radio and X-rays. Figure 1(3) shows the 2005 data. Fitting the data with a power law, we found $F_{\mathrm{R}} \propto F_{\mathrm{X}}^{0.71 \pm 0.03}$ and the fit is excellent. In other

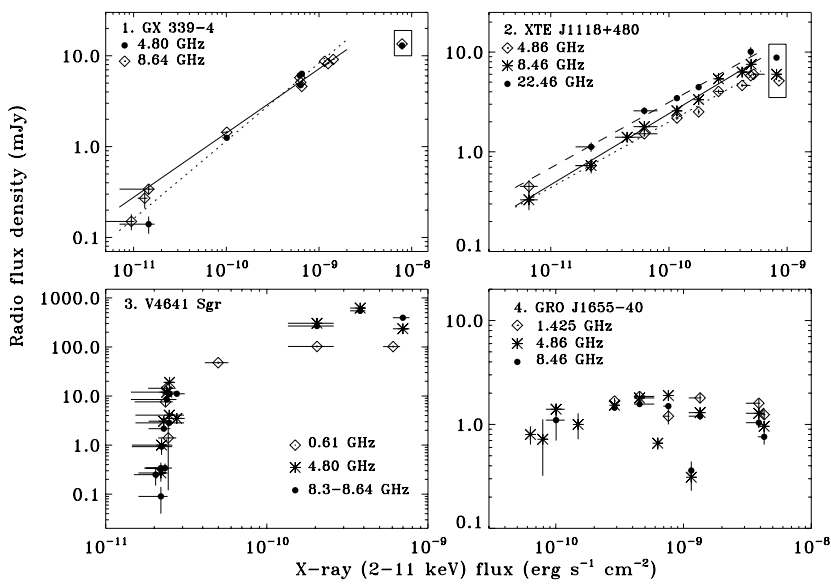

Fig. 2. The frequency dependence of radio/X-ray correlations. Radio data in various bands are shown for the selected sources. The data points in the box were taken during a different outburst (see text). Note that only data for the low-hard state are shown. (Panel 1) The best power-law fit to the $4.80 \mathrm{GHz}$ data is shown in the dotted line and to the $8.64 \mathrm{GHz}$ data in the solid line. (Panel 2) The best power-law fit to the $4.86 \mathrm{GHz}$ data is shown in the dotted line, to the $8.46 \mathrm{GHz}$ data in the solid line, and to the $22.46 \mathrm{GHz}$ data in the dashed line.

words, XTE J1118+480 happens to nicely follow the claimed universal radio/X-ray correlation over a flux range that spans roughly two orders of magnitude both in radio and X-rays. In fact, this is the tightest radio/X-ray correlation that has been seen among all microquasars.

V4641 Sgr is a member of the ever-growing population of fast X-ray transients. It was discovered during a giant outburst in 1999 that lasted only for a few days! During the outburst, the jets with superluminal motion were seen. The unusual characteristics of the source generated a lot of interests and consequently made it the target of a number of observational efforts. Unfortunately, there were no simultaneous/contemporaneous radio/X-ray observations during the most active period of the source, partly due to the briefness of the outburst. Figure 1(4) summarizes the results of all radio/X-ray measurements taken at nearly the end of this outburst and during smaller outbursts between 2002 and 2004, when the source was in the low-hard state. The radio and X-ray variabilities are again positively correlated but, taken at its face value, the correlation is quite complex in this case (see, however, discussion in the next section). At the low end of X-ray fluxes, the source hardly varied in X-rays while exhibited significant variability in the radio band. At higher fluxes, the two bands begin to show a tighter correlation. If we fit only the high-flux "branch" of the data set (i.e., the seven data points toward the high end of the fluxes), we would get $F_{\mathrm{R}} \propto F_{\mathrm{X}}^{1.14 \pm 0.01}$ (as indicated by the dotted line in the figure).

\subsection{Complexity of the radio/X-ray correlation in microquasars}

We proceeded to investigate possible spectral dependence of the observed radio/X-ray correlations. We selected sources in the sample for which multi-frequency radio data are available. Figure 2 summarizes the results. Like in Fig. 1, only data for the low-hard state are shown in Fig. 2.

As already mentioned, GX 339-4 is one of the two sources on which the claimed universal radio/X-ray correlation is based. The tight correlation is apparent in Fig. 2(1), as first reported by Corbel et al. (2003), using only the $8.64 \mathrm{GHz}$ data. We note, 
however, that we have added a new data point that was taken during a more recent outburst (in 2002) and that the new data point is clearly below the extrapolation of the reported correlation. The phenomenon is also evident for XTE J1118+480, as shown in Fig. 2(2), which now also includes data points from the 2000 outburst. There are two scenarios to consider. One is that the radio/X-ray correlation in these sources might level off at high fluxes; the other is that the normalization (or even the slope) of the correlation might be different for different outbursts. Unfortunately, the existing data are not sufficient to allow us to distinguish the two scenarios. In either case, the results have added a new twist to the radio/X-ray correlation in microquasars that any viable model must try to account for. It is also worth noting that the loose correlation in Cyg X-1 and the complex correlation in V4641 Sgr (see Fig. 1) might also be the manifestation of the same phenomenon.

Examining the radio/X-ray correlation in each radio band separately, we found that the $4.80 \mathrm{GHz}$ data can be best described as $F_{\mathrm{R}} \propto F_{\mathrm{X}}^{0.86 \pm 0.03}$ and the $8.64 \mathrm{GHz}$ data as $F_{\mathrm{R}} \propto F_{\mathrm{X}}^{0.71 \pm 0.01}$ for GX 339-4, when we excluded the 2002 data points. This result seems to suggest a spectral dependence of the radio/X-ray correlation, at least for GX 339-4 in the low-hard state. We repeated the analysis for XTE J1118+480, which also shows a tight power-law correlation. Excluding data from the 2000 outburst, we found that the $4.86 \mathrm{GHz}$ data can be best described as $F_{\mathrm{R}} \propto F_{\mathrm{X}}^{0.66 \pm 0.03}$, the $8.46 \mathrm{GHz}$ data as $F_{\mathrm{R}} \propto F_{\mathrm{X}}^{0.71 \pm 0.03}$, and the $22.46 \mathrm{GHz}$ data as $F_{\mathrm{R}} \propto F_{\mathrm{X}}^{0.66 \pm 0.07}$. Therefore, there is no apparent frequency dependence of the slope of the radio/X-ray correlation for XTE J1118+480, although there appears to be a shift in the overall normalization among different radio bands in this case. The phenomenology is, therefore, complex in this regard as well. For completeness, we also show, in Fig. 2, the multi-band radio measurements for GRO J1655-40 and V4641 Sgr. It is not obvious how to best quantify the radio/X-ray correlation in these cases. By eye, no frequency dependence of the correlation is apparent in either case.

\subsection{The radio/X-ray correlation beyond the low-hard state}

The claimed universal radio/X-ray correlation is said to be applicable mainly to the low-hard state of microquasars (Gallo et al. 2003). Unfortunately, the spectral states are, in general, not well defined, as discussed in great detail in Xue et al. (2006). The degree of arbitrariness in the definitions has made the discussion and comparison of results very difficult. Xue et al. (2006) suggested that the spectral states should be defined simply based on the overall shape of the SED. The low-hard state and high-soft state thus defined would, unfortunately, be only of theoretical value, because they are nearly impossible to catch in practice. These two diametrically-opposed states represent extreme scenarios in which only cold or hot accretion flows contribute to the X-ray emission from microquasars. So they might not even occur in nature. In this context, one can only speak of the quasi low-hard or high-soft state. We will do the same here.

Cyg X-1 and GX 339-4 have both shown strong anticorrelations between the radio and $\mathrm{X}$-ray variabilities during transitions between the low-hard and high-soft states (e.g., Braes \& Miley 1976; Corbel et al. 2000). To see whether this is a generic feature of microquasars, we carefully examined the data for the sources in our sample. Figure 3 summarizes the results for the transitional states, together with those for the low-hard state for comparison. We should emphasize that there is hardly any confusion between the low-hard state and transitional state in

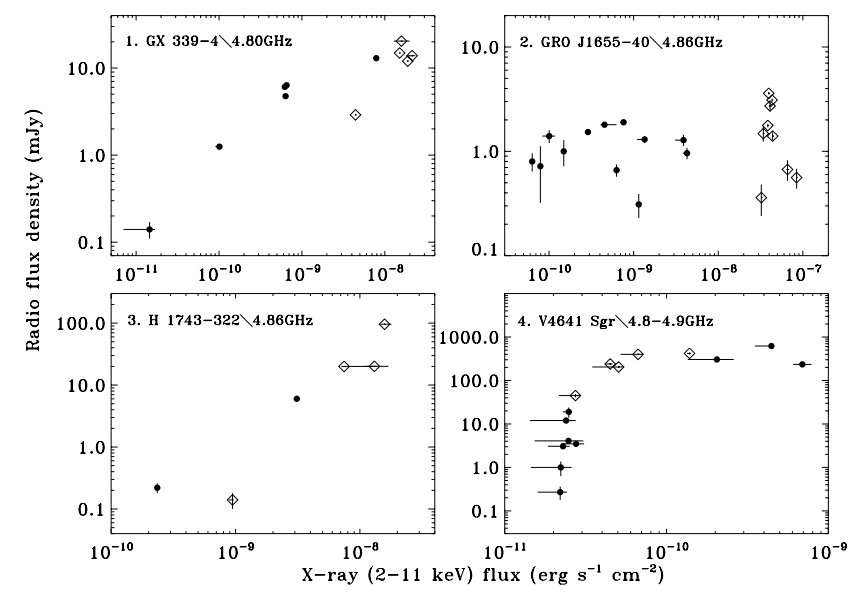

Fig. 3. The comparison of radio/X-ray correlations between the lowhard and transitional states. The low-hard state data are shown in filled symbols and the transitional state data in open symbols. Note that only the $4.8 \mathrm{GHz}$ data are shown here.

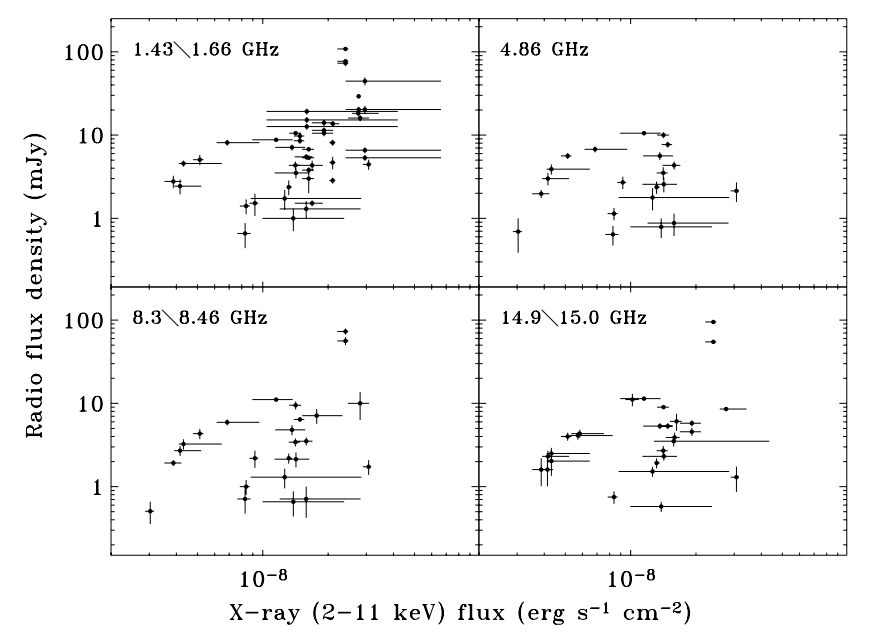

Fig. 4. The radio/X-ray correlation for XTE J1859+226 in the transitional state.

the definitions suggested by Xue et al. (2006), which we adopted for this work. For clarity, we included only the radio data taken at $\sim 4.8 \mathrm{GHz}$. There are two things worth noting. First of all, the flux ranges of the two states overlap, so the flux alone is not a reliable predictor of spectral states. This is not new (see, e.g., Homan et al. 2001) and is perhaps related to the spectral hysteresis associated with state transitions (e.g., Miyamoto et al. 1995; Zdziarski et al. 2004; Xue et al. 2006). Secondly, although the expected anti-correlation can be seen in GRO J1655-40 in the transitional state, the radio and X-ray variabilities seem to be positively correlated for other sources, including GX 339-4, during transitions, which is very puzzling. We caution, however, against drawing any definitive conclusions, given the sparseness and the very limited dynamical ranges of the data.

Further complication arises when we examine the behavior of XTE J1859+226 in the transitional state, as shown in Fig. 4. The "loop" pattern shows the lack of one-to-one correspondence between the X-ray and radio fluxes, which seems to indicate the presence of some kind of hysteresis associated with the variabilities of the source between the X-ray and radio bands. The phenomenon persists across all radio bands. The data were derived from observations of XTE J1859+226 during its 1999 outburst. 
The outburst follows a fast-rise-slow-decay profile. Looking at the data more carefully, we found that the "loop" did not vanish after we excluded data from the rising phase of the outburst. Therefore, the phenomenon is not related to any hysteresis that might be associated with the rise and fall of the source during the outburst.

\section{Discussion}

We have critically examined the radio/X-ray correlation for microquasars. We have clearly demonstrated that there is not a universal relationship that can account for the correlated variabilities of microquasars between the radio and X-ray bands, even for the low-hard state. In fact, the observed radio/X-ray correlation cannot be adequately described by a simple power law in most cases that we have examined. Our results are, therefore, at odds with those of Gallo et al. (2003). We believe that the results are quite robust, given that they are based on a sample that includes representatives of various sub-populations of microquasars. On the other hand, there is, to varying degrees, a general positive correlation between the radio and X-ray variabilities among the sources in our sample. In one case (XTE J1118+480), the correlation is very tight and follows nicely the claimed universal correlation. Such diverse behaviors present a severe challenge to our understanding of the phenomenon.

Besides the lack of universality of the radio/X-ray correlation in microquasars, we also found further complications, some of which might be generic and some might reflect the personality of individual sources. Specifically, there seems to be some spectral dependence of the radio/X-ray correlation, at least for GX 339-4. Therefore, even for a given source, one might need to specify the spectral bands of interest when one speaks of the correlation quantitatively. On the other hand, no such spectral dependence is apparent for XTE J1118+480, which shows an equally tight (if not tighter) power-law correlation between the radio and X-ray bands. So, this is probably not a general phenomenon for all microquasars. Another feature that is worth noting is the possibility that the radio/X-ray correlation might exhibit quantitative difference between different outbursts of the same source. However, the existing data do not allow a definitive conclusion on the issue.

Examining the data from observations of microquasars in the transitional state, we seem to confirm the presence of anti-correlation between the radio and X-ray fluxes for GRO J1655-40, which has been seen previously. However, for other sources, the radio/X-ray correlation appears to be positive, qualitatively similar to the low-hard state, which is puzzling. However, the dynamical range of the data is limited in all cases, so these results should be taken with a grain of salt. It has been speculated that for microquasars the jets might be steady in the low-hard state but consist of discrete ejection events during the transitions (e.g., Corbel et al. 2000). If this is the case, it would not be surprising that the radio/X-ray correlation would be, at least quantitatively, different between the low-hard state and the transitional state. From a theoretical point of view, even if the correlation reflects a physical coupling between the jets and accretion flows, the details of the coupling would not remain the same due, e.g., to the changes in the accretion flows during state transitions. More data are clearly needed to reveal and ultimately quantify the difference in the correlation between the states.

Finally, it is puzzling that the $\log F_{\mathrm{R}}-\log F_{\mathrm{X}}$ plot for XTE J1859+226 in the transitional state shows a loop pattern. This certainly adds another level of complication to the discussion of the radio/X-ray correlation in microquasars.
The phenomenon might be an indication of some kind of hysteresis but the hysteresis cannot be associated with the rising and decaying phases of outbursts. It remains to be seen whether the phenomenon also occurs in other microquasars.

Acknowledgements. This work has made use of data obtained through the High Energy Astrophysics Science Archive Research Center Online Service, provided by the NASA/Goddard Space Flight Center. We gratefully acknowledge financial support from NASA and from the Purdue Research Foundation.

\section{References}

Arnaud, K. A. 1996, Astronomical Data Analysis Software and Systems V, ed. G. Jacoby, \& J. Barnes, ASP Conf. Ser., 101, 17

Blackburn, J. K. 1995, in Astronomical Data Analysis Software and Systems IV, ed. R. A. Shaw, H. E. Payne, \& J. J. E. Hayes (San Francisco: ASP), ASP Conf. Ser., 77, 367

Braes, L. L. E., \& Miley, G. K. 1976, Nature, 264, 731

Brocksopp, C., Corbel, S., Rupen, M., et al. 2005, The Astronomer's Telegram, 612

Brocksopp, C., Fender, R. P., McCollough, M., et al. 2002, MNRAS, 331, 765 Chaty, S., Haswell, C. A., Malzac, J., et al. 2003, MNRAS, 346, 689 Choudhury, M., Rao, A. R., Vadawale, S. V., \& Jain, A. K. 2003, ApJ, 593, 452 Corbel, S., Fender, R. P., Tzioumis, A. K., et al. 2000, A\&A, 359, 251 Corbel, S., Nowak, M. A., Fender, R. P., et al. 2003, A\&A, 400, 1007 Cui, W. 2004, ApJ, 605, 662

Dhawan, V., Pooley, G. G., Ogley, R. N., \& Mirabel, I. F. 2000, IAU Circ., 7395, 2

Dickey, J. M., \& Lockman, F. J. 1990, ARA\&A, 28, 215

Esin, A. A., McClintock, J. E., Drake, J. J., et al. 2001, ApJ, 555, 483

Falcke, H., \& Biermann, P. L. 1995, A\&A, 293, 665

Fender, R. P., Hjellming, R. M., Tilanus, R. P. J., et al. 2001, MNRAS, 322, L23 Frontera, F., Zdziarski, A. A., Amati, L., et al. 2001, ApJ, 561, 1006 Gallo, E., Fender, R. P., \& Pooley, G. G. 2003, MNRAS, 344, 60 Gallo, E., Corbel, S., Fender, R. P., et al. 2004, MNRAS, 347, L52 Gleissner, T., Wilms, J., Pooley, G. G., et al. 2004, A\&A, 425, 1061 Heinz, S. 2004, MNRAS, 355, 835

Hjellming, R. M., Rupen, M. P., Hunstead, R. W., et al. 2000, ApJ, 544, 977 Homan, J., Wijnands, R., van der Klis, M., et al. 2001, ApJS, 132, 377 Hynes, R. I., Mauche, C. W., Haswell, C. A., et al. 2000, ApJ, 539, L37 Ishwara-Chandra, C. H., \& Pramesh Rao, A. 2005, ChJAA, 5, 269 Kalemci, E., Tomsick, J. A., Rothschild, R. E., et al. 2006, ApJ, 639, 340 Liang, E. P. 1998, Phys. Rep., 302, 67

Markoff, S., Falcke, H., \& Fender, R. 2001, A\&A, 372, L25

Markoff, S., Nowak, M., Corbel, S., et al. 2003, A\&A, 397, 645

McClintock, J. E., Haswell, C. A., Garcia, M. R., et al. 2001, ApJ, 555, 477 Meier, D. L. 2001, ApJ, 548, L9

Mirabel, I. F., \& Rodríguez, L. F. 1999, ARA\&A, 37, 409

Miyamoto, S., Kitamoto, S., Hayashida, K., \& Egoshi, W. 1995, ApJ, 442, L13 Robertson, S. L., \& Leiter, D. J. 2004, MNRAS, 350, 1391

Rupen, M. P., Mioduszewski, A. J., \& Dhawan, V. 2003a, IAU Circ., 8105, 3 Rupen, M. P., Mioduszewski, A. J., \& Dhawan, V. 2003b, The Astronomer's Telegram, 139

Rupen, M. P., Mioduszewski, A. J., \& Dhawan, V. 2003c, IAU Circ., 8112 Rupen, M. P., Mioduszewski, A. J., \& Dhawan, V. 2003d, The Astronomer's Telegram, 172

Rupen, M. P., Dhawan, V., \& Mioduszewski, A. J. 2003e, The Astronomer's Telegram, 175

Rupen, M. P., Dhawan, V., \& Mioduszewski, A. J. 2004a, The Astronomer's Telegram, 303

Rupen, M. P., Mioduszewski, A. J., \& Dhawan, V. 2004b, The Astronomer's Telegram, 314

Rupen, M. P., Mioduszewski, A. J., \& Dhawan, V. 2005, The Astronomer's Telegram, 575

Senkbeil, C., \& Sault, B. 2004, The Astronomer's Telegram, 302

Shaposhnikov, N., Swank, J., Shrader, C. R., et al. 2007, ApJ, 655, 434

Uemura, M., Kato, T., Ishioka, R., et al. 2004, PASJ, 56S, 61U

Wilms, J., Nowak, M. A., Pottschmidt, K., et al. 2006, A\&A, 447, 245

Xue, Y. Q., \& Cui, W. 2005, ApJ, 622, 160

Xue, Y. Q., Wu, X.-B., \& Cui, W. 2006 [arXiv: astro-ph/0606194]

Yuan, F., \& Cui, W. 2005, ApJ, 629, 408

Yuan, F., Cui, W., \& Narayan, R. 2005, ApJ, 620, 905

Zdziarski, A. A., Lubiński, P., Gilfanov, M., \& Revnivtsev, M. 2003, MNRAS, 342,355

Zdziarski, A. A., Gierliński, M., Mikolajewska, J., et al. 2004, MNRAS, 351, 791 